$81 \mid 2012$

Pouvoirs du mythe dans les littératures francophones du Maghreb et du Machrek

\title{
De Didon à Elissa : la réappropriation d'un mythe
}

\section{Abdelkader Amri}

\section{OpenEdition}

\section{Journals}

Édition électronique

URL : http://journals.openedition.org/recherchestravaux/545

DOI : $10.4000 /$ recherchestravaux.545

ISSN : 1969-6434

Éditeur

UGA Éditions/Université Grenoble Alpes

Édition imprimée

Date de publication : 30 décembre 2012

Pagination : 65-74

ISBN : 978-2-84310-238-7

ISSN : 0151-1874

\section{Référence électronique}

Abdelkader Amri, « De Didon à Elissa : la réappropriation d'un mythe », Recherches \& Travaux [En ligne], 81 | 2012, mis en ligne le 30 juin 2014, consulté le 08 septembre 2020. URL : http://

journals.openedition.org/recherchestravaux/545; DOI : https://doi.org/10.4000/recherchestravaux. 545 
Abdelkader AmRI

Université Stendhal - Grenoble 3

\section{De Didon à Elissa : la réappropriation d'un mythe}

À côté de la tradition arabo-musulmane, plusieurs civilisations vivent dans le conscient et l'inconscient des Tunisiens : la berbère, la punique, la romaine, la byzantine, la vandale, l'andalouse, l'ottomane et la française. Ces différents apports ont marqué le pays et représentent actuellement sa richesse intellectuelle : le Tunisien d'aujourd'hui se sent le descendant de toutes ces cultures qui façonnent son identité plurielle. Ce sentiment de continuité avec des ères antéislamiques est fréquent dans le pays, à tous les échelons de la société, partagé par la majorité des habitants qui manifestent leur amour pour ces ères anciennes. Les personnages phéniciens ou romains tels qu'Elissa, Hannibal, Hamilcar, Hasdrubal et d'autres encore occupent une place importante dans la publicité, dans le domaine touristique, sur les enseignes des magasins et dans les noms d'établissements publics. Ce retour à un temps révolu et cette nostalgie d'une époque passée correspondent à l'affirmation d'une fierté nationale qui se développe en association avec l'héritage musulman, bien évidemment. Une telle diversité ne manque pas d'influencer la création littéraire. Les cultures anciennes inspirent les romanciers contemporains et, en particulier, les romanciers francophones, qui par le choix de leur langue d'expression, symbolisent le brassage des héritages en Tunisie. Nombre d'entre eux retracent ainsi la vie d'un roi, d'un guerrier ou d'un personnage mythique empruntés à des ères culturelles différentes du monde arabomusulman. C'est le cas de Fawzi Mellah dans Elissa la reine vagabonde ${ }^{\mathrm{I}}$ et de Sophie El Goulli dans Hashtart à la naissance de Carthage 2 .

I. F. Mellah, Elissa la reine vagabonde, Seuil, i988. Les références de pages sont données entre parenthèses après la citation.

2. Sophie El Goulli, Hashtart, à la naissance de Carthage, Tunis, Cérès, 2004. Les références de pages sont données entre parenthèses après la citation. 
Le roman de Fawzi Mellah se présente comme un ensemble de lettres écrites par Elissa à son frère Pygmalion, qui l'a évincée du royaume après avoir tué son mari Acherbas. Elle raconte son voyage, depuis son départ de son pays de Tyr, jusqu'au jour où elle va s'immoler pour ne pas épouser le roi numide Iarbas. Elle décrit minutieusement les différentes étapes de son errance, sa relation avec son équipage et avec les autochtones, ses escales, ses départs et sa ruse avec Iarbas pour construire qart Hadasht, la cité nouvelle. Ses lettres sont pleines d'enseignements. Elle réfléchit sur le sacrifice, la trahison, la beauté, la mer, la musique, la différence entre les sexes et l'art de gouverner. Dans cette œuvre, Fawzi Mellah se présente comme le traducteur de stèles héritées de son grand père et dont le déchiffrement correspond aux différentes lettres.

Quant à Sophie El Goulli, dans Hashtart, à la naissance de Carthage, elle aborde l'histoire d'Elissa à travers celle d'Hashtart, sa fille adoptive. D'une manière rétrospective, la jeune héroïne relate l'histoire d'Elissa, en mettant en évidence ses qualités en tant que femme et gouvernante, retrouvant ainsi les réflexions de Fawzi Mellah. Hashtart raconte son histoire depuis la fuite de Tyr avec Elissa, jusqu'au jour où la reine s'immole par le feu, lors de son mariage avec Iarbas, le roi Numide. Cet événement douloureux pousse la petite Hashtart à quitter le palais royal, sans que personne ne se rende compte de son absence. Après une longue errance, elle perd connaissance dans la forêt. Trouvée par des bergers, elle est amenée au palais royal où on prend soin d'elle jusqu'à sa convalescence. Les années passent : Hashtart grandit et sa beauté fascine le roi larbas qui ne peut s'empêcher de se marier avec elle. Ainsi, Hashtart réalise-t-elle ce que sa reine Elissa n'a pas pu réaliser. Son mariage unit les Numides aux Phéniciens.

Fawzi Mellah et Sophie El Goulli ont pris comme héroïne la reine phénicienne Elissa, plus connue en Occident sous le nom de Didon, la célèbre héroïne de Virgile (L'Énéide) ou de Berlioz (Les Troyens). À travers l'évocation de cette femme légendaire, les écrivains manifestent un double objectif: d'une part, ils revendiquent leur passé antéislamique, s'inscrivant dans la continuité de leurs aïeux phéniciens, d'autre part, ils se réapproprient une figure mythique de la culture gréco-latine pour la réinscrire dans leur histoire, relativisant - sans jamais les renier - les cultures dominantes apportées par les grands courants de colonisation. Le choix d'une reine phénicienne, le glissement de Didon à Elissa pour nommer l'hérö̈ne mythique, l'absence des Troyens, d'Énée et de la passion amoureuse, affirment haut et fort ce double mouvement d'affranchissement et de sereine revendication. En retraçant l'itinéraire d'Elissa, en choisissant une figure mythique, Fawzi Mellah et Sophie El Goulli établissent une généalogie prestigieuse qui satisfait chez eux «une violente demande d'être $»$ ».

3. L. Ibrahim Ouali, «La ville en ruines dans le roman maghrébin francophone et arabophone», 


\section{Pour une identité méditerranéenne}

Les deux auteurs, originaires de zones côtières, accordent dans leurs œuvres une grande importance à la mer (Fawzi Mellah est né à la Marsa, Sophie El Goulli à Sousse). En la plaçant au cœur des événements et des affects, ils l'érigent en «thème identitaire dominant ${ }^{4} »$. Mais l'identité qu'ils revendiquent ne renvoie nullement au "caractère de ce qui est un ${ }^{5}$ ». En effet :

comment peut-on être UN quand l'individu appartient à un lieu géographique dont les rivages méditerranéens ont été toujours occupés par divers peuples dont les langues, religions, civilisations sont différentes de celles de l'autochtone ${ }^{6}$ ?

Chez Fawzi Mellah, la mer est présentée comme le lieu propice à la diversité, le lieu où les couleurs changent et s'entrelacent pour exprimer la beauté $\mathrm{du}$ monde, pour dire la splendeur des éléments. Ces différentes couleurs peuvent se lire, métaphoriquement, comme les différentes civilisations qui ont enrichi l'histoire et participent à la séduction du monde méditerranéen : «Nous vîmes le soleil qui enveloppait les traits mauves de la mer. L'aube virait du mauve au rouge violacé, puis du violet à l'éclat doré» (Mellah, p. IO2). Sophie El Goulli se montre, elle aussi, sensible à l'extraordinaire richesse culturelle symbolisée par la mer :

Rien que la mer tout autour, la mer à l'infini [...] la mer est si belle, si calme. Au cours de ce long voyage, elle lui découvrait, à la mer, d'autres visages et de terribles fureurs qui ne l'effrayaient jamais. Elle la connaissait bien la mer. Une amie à qui elle parle en se promenant le long de la plage. À qui elle se confie. C'est que leurs vies se confondent. Tous ses souvenirs la contiennent. (El Goulli, p. I4)

Cette mer, avec toutes les civilisations qu'elle a abritées, est devenue un lieu de régénérescence pour Hashtart, qui ne se prive jamais de venir méditer devant une telle beauté (la scène est récurrente dans le roman) :

Tôt, elle se réveillait. Très tôt. En toutes saisons, pour assister au lever de l'Astre-

Dieu. Ses rayons qui embrassaient le ciel, la mer et la terre de pourpre violet, rose turquoise et d'or, elle aimait les sentir la pénétrer. (El Goulli, p. I2)

La contemplation de la mer renvoie naturellement à une forte revendication culturelle et politique. Comme le note Hashtart, la reine se distingue

dans La Mémoire en ruines, V.-A. Deshoulières et P. Vacher (dir.), Clermont-Ferrand, CRLMC, 2000, p. 166.

4. D. Abassi, Quand la Tunisie sinvente. Entre Orient et Occident, des imaginaires politiques, Éditions Autrement, 2009, p. 7.

5. Robert, Dictionnaire de langue française, 2007.

6. B. Alia Bournaz, Essais sur la littérature tunisienne d'expression française, Louvain-la-Neuve, Bruylant-Académia, 2005, p. I6. 
de la foule, qui se montre incapable de respirer à la même hauteur qu'elle : "Dans l'air dénaturé par la respiration de cette foule muette flotte le parfum iodé de la mer. Seule la reine le sent et s'en remplit le cœur.» (El Goulli, p. 25) Le symbolisme est clair : quand la culture dominante tourne le dos à la Méditerranée, Sophie El Goulli, en se projetant dans son héroïne mythique, refuse de se laisser enfermer dans une identité unique. Seule la mer et la diversité qu'elle représente lui procure un sentiment d'émancipation, qui est totalement absent dans la culture islamique dominante - dans la culture islamique comme domination.

La rupture avec ces ères anciennes imposée par l'islam est compensée par le spectacle de la Méditerranée, car comme le soutient Fawzi Mellah : "La mer m'introduisait au royaume sans frontières» (Mellah, p. 30). À travers l'errance («l'errance seule m'attirait», Mellah, p. 29), le romancier cherche à «reconstituer la Phénicie ailleurs que la Phénicie» (Mellah, p. 3), c'est-àdire qu'il veut la retrouver dans la Tunisie où il vit, une Tunisie où l'eau et la terre, le rêve et la réalité, les racines et les voyages se combineraient harmonieusement : «si la mer fait rêver, la terre fait vivre» (Mellah, p. 7), «la mer et la terre se compénètrent sans se déchirer, il est bon que la mer et la terre se serre sans s'étouffer" (Mellah, p. 17). La diversité culturelle que représente la Méditerranée se matérialise ainsi dans la présence de plantes importées de lieux différents : le datura est français, l'hibiscus est une plante tropicale, le caroubier est méditerranéen, les orangers et les citronniers sont des plantes apportées en Tunisie par les Andalous. Elles s'unissent toutes malgré leurs origines différentes pour parfumer et donner une "odeur si chère» (El Goulli, p. Io) à cette mer Méditerranée :

Une erreur de navigation nous poussa vers une colline parfumée. De loin nous n'apercevions aucune trace humaine, mais une verte avalanche d'arbres odorants, orangers, citronniers, figuiers caroubiers, cyprès, genévriers, hibiscus, daturas et sycomores faisaient mine de se précipiter à l'eau comme pour mieux embaumer la mer. (Mellah, p. II4)

Loin de la monoculture, «l'identité a la mémoire pour socle et la continuité pour litière» (Mellah, p. 99). À la discontinuité qu'ils déplorent dans la culture islamique dominante, répond la continuité du lien avec la Méditerranée.

\section{Le sexe : «une curieuse manie de qualifier les êtres» (Mellah, p. 152)}

Le culte de la mer, la volonté de valoriser les cultures méditerranéennes sont à lire, non comme le refus de l'islam, mais comme l'apologie d'un syncrétisme culturel porteur d'harmonie et de paix. En choisissant une héroïne venue 
d'ailleurs (la Phénicie), les deux romanciers établissent des liens heureux entre le Maghreb et le Machrek. Mais il n'est naturellement pas indifférent que le choix des écrivains tunisiens se porte sur une femme, le choix du sexe n'étant pas fortuit. Depuis l'indépendance en 1956 et sous l'impulsion de Bourguiba, la Tunisie se distingue de la plupart des pays arabes par une politique audacieuse en direction des femmes. C'est cet apport et cet héritage que Sophie El Goulli et Fawzi Mellah ne cessent de célébrer et de protéger en choisissant d'élire un personnage comme Elissa, cette figure plus mythique qu'historique, qui exalte la capacité des femmes à sortir de la maison et à gouverner l'État.

La condition féminine ne cesse de préoccuper nos deux auteurs qui ont choisi des figures antiques féminines afin de les comparer avec la femme dans la culture musulmane actuelle. Lors du passage de l'ère antéislamique à l'ère islamique, la femme a perdu bon nombre de ses droits. Les auteurs ne manquent pas de focaliser l'attention sur la bizarre habitude de qualifier les êtres selon leur sexe et de fonder la société sur une séparation draconienne du rôle de chacun d'eux. Fawzi Mellah déplore ainsi que la femme soit considérée comme un être second. Dans l'héritage, par exemple, elle n'a droit qu'à la moitié de la part de l'homme, conformément à la sourate "Les femmes» du Coran. Quant à la vie politique, la femme est dépourvue du droit de gouverner ou de diriger un groupe d'hommes'.

Le passage de la femme "païenne» à la femme musulmane est donc perçu par les deux auteurs comme une régression. Selon Fawzi Mellah, «les plus grandes calamités $[. .$.$] seraient liées à la chute du matriarcat et aux arrogances$ du patriarcat» (Mellah, p. 24). C'est pour cette raison qu'Elissa est en quête d'une

terre inculte sur laquelle nos mains sculpteraient l'esprit et donneraient corps à la beauté. Une terre fragile et maternelle. Pas une patrie (et qui a inventé ce terme insensé qui évoque le père!) C'était une matrie que je désirais. Je l'inventerais, s'il le fallait. (Mellah, p. 82)

Les deux auteurs rêvent à la femme phénicienne, décrite comme une femme autonome, ayant le droit d'aborder tous les sujets, de se conduire à égalité avec les hommes. Ils revendiquent que la femme tunisienne et musulmane sache parler et convaincre, tout comme Elissa qui raisonne les prêtres et exige «Qu’une figure féminine siégeât également dans les sanctuaires de [sa]

7. J. Berque, Le Coran. Essai de traduction, Albin Michel, 2002, t. IV, p. 34 (sourate «Les femmes») : "Les hommes assument les femmes à raison de ce dont Dieu les avantage sur elles et de ce dont ils font dépense sur leurs propres biens. Réciproquement, les bonnes épouses sont dévotieuses et gardent dans l'absence ce que dieu sauvegarde. Celles de qui vous craignez l'insoumission, faites-leur la morale, désertez leur couche, corrigez-les. Mais une fois ramenées à l'obéissance, ne leur cherchez pas prétexte.» 
ville nouvelle» (Mellah, p. 136). Ils espèrent lever le voile sur le langage de la femme comme on a voulu l'enlever de son corps. Sophie El Goulli dénonce l'oppression qui a maintenu cachée la voix féminine ("ce mutisme l'effraie", El Goulli, p. 2I) et, pour lutter contre ce silence imposé, la romancière prend la parole en imposant son désir de s'exprimer par l'écriture, c'est-à-dire par une voix qui porte bien au-delà de l'espace confiné de la maison. Comme le remarque Marta Segarra dans son analyse de romans écrits par des femmes arabes, le "désir de narrativité y est en effet extrêmement marqué par la volonté de combattre le silence ancestral des femmes, de prendre la parole et de s'exprimer par elle-même ${ }^{8}$ ".

Pour un lecteur occidental, la figure de Didon est principalement reçue comme une figure de reine amoureuse. La puissante reine de Carthage touche moins par la grandeur et la sagesse de son pouvoir que par la violence d'une passion qui la conduit à s’immoler par le feu quand Énée, appelé par son devoir, quitte Carthage pour reprendre la mer vers l'Italie. Dans l'imaginaire occidental, Didon s'impose comme la reine redevenue femme, comme la supériorité d'une passion privée sur la fonction sacrée de la souveraine. Rien de tel dans les deux romans tunisiens : en choisissant de raconter la fondation de Carthage, en évoquant longuement la sagesse politique d'Elissa, Sophie El Goulli et Fawzi Mellah ont choisi de renouveler la grande figure mythique de Didon en la rendant à son statut de reine, en passant d'une exemplarité sentimentale à une exemplarité politique. En transformant Didon en Elissa, les deux romanciers changent la portée du mythe : quand Virgile et Berlioz chantaient la douleur de l'amour, El Goulli et Mellah font remonter l'hérö̈ne sur le trône et lui rendent la source principale du pouvoir : la parole.

Mais que faire de la parole quand on n'est pas une reine phénicienne? Comment faire de ce mythe un exemple que chacun adapte à sa manière, selon sa situation? Sophie El Goulli et Fawzi Mellah ont choisi de faire revivre cette parole par le biais de la littérature; si la littérature restaure les voix perdues et donne voix aux sans voix, les deux romans tunisiens font entendre une voix perdue qui rappelle à chacun que le silence imposé aux femmes est une réalité historique et que toute réalité historique n'a rien de définitif. La voix littéraire des romanciers tunisiens redonne vie à la parole passée et, ressuscitant le mythe de la femme libre, appelle chacun de nous - et tout particulièrement les femmes - à prendre la parole à son tour. Prendre la parole pour chanter les couleurs de la vie, heureuses ou malheureuses, en accord avec les couleurs changeantes de la mer...

8. M. Segarra, Leur pesant de poudre, romancières francophones du Maghreb, L'Harmattan, I997, p. 27. 


\section{Les couleurs et la musique de la vie}

Elissa se voue à la beauté et à l'élégance. Femme et souveraine, la reine a l'habitude de s'habiller somptueusement. Elle accorde une grande importance à la couleur pourpre, symbole de la Phénicie et aux bijoux qui marquent son rang. Pendant les moments de deuil, la couleur pourpre se change en couleur blanche, telle un suaire, et la reine se coupe les cheveux pour s'enlaidir. La scène de rasage est récurrente durant l'errance de l'héroïne mythique : "Je demandai que l'on me coupât de nouveau les cheveux» (Mellah, p. 27). Avant de fuir Tyr ${ }^{9}$ et en entendant les conseils des sénateurs, elle est « habillée de blanc, comme il sied à une reine endeuillée» (Mellah, p. 27). De même, quand sur le bateau, "Nous eûmes à subir les coups de la mort [...] je mis une robe blanche et je m'enfermai dans les soutes» (Mellah, p. 96). Même le jour de son immolation, elle n'oublie pas d'accorder une importance à ce qu'elle va porter et finit par choisir la tenue qui convient à cet événement :

Aujourd'hui, c'est vêtue de lin blanc et non de la pourpre royale qu'elle se veut.

Aujourd'hui point de bijoux mais l'anneau que son époux défunt lui a offert pour sceller leur union et qu'elle n'a jamais ôté. Aujourd'hui, seul signe de sa haute naissance et de son rang, un bandeau de soie tressé de pourpre et d'or. (Mellah, p. 23)

Fawzi Mellah décrit cette figure mythique dans deux moments opposés : le deuil et la joie. À partir de cette dualité des couleurs, il met en exergue la beauté de cette reine et ne peut pas cacher sa fascination pour elle. Pour le deuil comme pour la joie, la couleur est gaie. C'est un contraste implicite que crée l'auteur avec la culture islamique, dans laquelle la femme est condamnée à se vêtir en noir, en se couvrant de la tête jusqu'aux pieds afin d'effacer toutes les traces de séduction que peut dégager son corps ${ }^{\text {10 }}$. Hashtart, la fille adoptive d'Elissa, aime aussi la beauté et accorde une grande importance à sa tenue, conformément à l'éducation qu'elle avait reçue de sa mère dès son plus jeune âge. La jeune fille assiste fascinée à l'habillement des sœurs d'Iarbas :

Un dernier bain, symbolique, puis commença la cérémonie de l'habillement. Une tunique aérienne, droite toute blanche, une autre tunique plus lourde, blanche

9. Évincée du royaume auquel elle avait droit, Elissa fuit Tyr de peur d'être tuée.

IO. J. Berque, Le Coran, essai de traduction, ouvr. cité, XXIV, 3I : "Dis aux croyantes de baisser les yeux et de contenir leur sexe; de ne pas faire montre de leurs agréments, sauf ce qui en émerge, de rabattre leur fichu sur les échancrures de leur vêtement. Elles ne laisseront voir leurs agréments qu’à leur mari, à leurs enfants, à leurs pères, beaux-pères, fils, beaux-fils, frères, neveux de frères ou de sœurs, aux femmes (de leur communauté), à leurs captives, à leurs dépendants hommes incapables de l'acte, ou garçons encore ignorants de l'intimité des femmes. Qu'elles ne piaffent pas pour révéler ce qu'elles cachent de leurs agréments par-dessus tout repentez vous envers Dieu, vous tous les croyants, dans l'espoir d'être des triomphants». 
aussi mais émaillée de broderies de couleurs vives. Une troisième tunique plus courte, en tissu épais, à fines bandes de couleurs vives brodées d'or. Un grand carré de toile bariolée couvrait les cheveux finement tressés et parfumés et retombait sur les épaules. Des boucles d'oreille, des colliers des anneaux de pied en or incrusté de pierre rouge vert et turquoise brillaient et tintaient à chaque mouvement. Fascinée, Hashtart suivait les mains habiles qui s’affairaient sur les visages des épouses. On l'habilla, elle aussi, on la para elle aussi. (El Goulli, p. 89)

La couleur a également beaucoup d'importance chez Sophie El Goulli : le blanc, neutre, les couleurs vives et bigarrées, le rouge, le vert et le turquoise s'associent pour illustrer la joie et exprimer la beauté de la femme. Le soin accordé aux cheveux et aux bijoux est très présent chez elle aussi, car se faire belle est le souci de toutes les femmes, comme s'il s'agissait de concilier le pouvoir perçu comme masculin et la beauté réservée aux femmes. Sophie El Goulli rejoint Fawzi Mellah dans ses allusions à la situation présente : elle insiste comme lui sur le port des tuniques, ce vêtement ajusté sur le corps qui laisse deviner les formes. Porter des tuniques était permis chez les Phéniciens, montrer la beauté corporelle de la femme à travers ses habits était tout ce qu'il y avait de plus normal. Dans la culture islamique, la femme rompt avec cette coutume et ne peut mettre que des vêtements amples qui cachent la beauté de ses formes.

Sophie El Goulli décrit la cérémonie d'habillement à la manière des mariages tunisiens, surtout les mariages du Sahel, la région où elle vit. Cet anachronisme volontaire peut s'expliquer de deux manières. Selon une première hypothèse, l'auteur veut montrer que la femme tunisienne est différente des autres femmes musulmanes obligées de mettre le voile. Elle veut rappeler que la femme en Tunisie est libre dans un pays libre. Selon une deuxième hypothèse, la liberté dont jouit la femme tunisienne actuellement remonterait à une ère très ancienne, celle des Phéniciens. Ainsi El Goulli, s'intéresse-t-elle à la frontière qu'a imposée l'islam par rapport aux civilisations qui l'ont précédé.

L'amour qu'éprouve Elissa pour la beauté de son corps et de sa tenue vestimentaire n'est qu'un reflet de la sensibilité de son âme. Cette âme, encline à aimer tout ce qui est beau et artistique, apprécie la musique comme un moyen de communication entre Dieu et son peuple. Elissa découvre l'absence de musique à Sabrata, la ville Libyenne où elle a débarqué après avoir quitté Chypre, comme un phénomène étonnant : "Ce qui me surprenait le plus, [...] c'était l'absence totale de la musique» (Mellah, p. 56) - cette absence étant ressentie comme l'absence d'un dialogue entre le Dieu et son peuple : «Peut-on imaginer un peuple sans musique? Ce serait comme un peuple auquel Dieu ne parlerait pas» (Mellah, p. 55). Et c'est grâce à cette reine que le peuple de Sabrata renoue les liens avec son Dieu, c'est à travers la musique 
jouée par les Phéniciens que le dialogue s'instaure à nouveau entre le ciel et la terre : "C'était Dieu qui par mes musiciens parlait au peuple de Sabrata" (Mellah, p. 56).

Or cet échange grâce à la musique s'éclipse dans la culture islamique qui considère souvent l'art des sons comme une jouissance interdite, une illusion ou un abus, compte tenu de l'aspect éphémère de la vie "d'ici-bas". Dans la sourate «Le fer ${ }^{\mathrm{II}}$ », une menace se profile envers ceux qui profitent des délectations et des plaisirs de la vie au détriment de la religion. Chez les Phéniciens, au contraire, la musique se jouait entre hommes et femmes. Les mêmes préoccupations se retrouvent chez Sophie El Goulli. En parlant d'une fête "païenne», elle met en exergue la rupture et les interdictions imposées par la culture islamique, en particulier en ce qui concerne la séparation des sexes, à l'opposé de ce qui se passait dans les fêtes phéniciennes où «les hommes sortent de leurs maisons et entonnant les chants rituels [...] les femmes leur répondant» (El Goulli, p. 93). De la musique, on passe tout naturellement à la danse dans ces fêtes harmonieuses où «des danseuses entrent dans le cercle. Des danseurs viennent les rejoindre. Danses d'amour heureux ou malheureux. Danses de séduction qui échauffent le cœur et le corps» (El Goulli, p. 94). Cette joie partagée entre l'homme et la femme ne s'oppose pas à la croyance religieuse puisque "[les dieux] sont satisfaits qui assistent sûrement et protègent» (El Goulli, p. 94).

En redonnant vie à une figure mythique longtemps associée au monde latin et occidental, Sophie El Goulli et Fawzi Mellah nous rappellent que le premier pouvoir du mythe est de circuler. Partie de Phénicie, Elissa-Didon a pris la mer pour errer de pays en pays avant de s'établir en Afrique du Nord, de fonder Carthage et de diriger un royaume prospère. Partis d'une lecture de Virgile, les deux romanciers tunisiens se sont réapproprié un mythe dont ils déploraient la lecture strictement européenne. Mais si leur démarche se veut volontaire, voire revendicatrice (au meilleur sens du terme), leur projet n'est jamais animé d'un désir de rupture. Profondément marqués par la culture française, écrivains de langue française, Sophie El Goulli et Fawzi Mellah ne cherchent pas à exclure l'apport occidental, même si cet apport est souvent associé à la violence coloniale. Pour eux, comme pour tant de Tunisiens, voire de Maghrébins, l'héritage européen fait partie intégrante de la Tunisie

II. J. Berque, Le Coran, essai de traduction, ouvr. cité, LVII, 20 : «Sachez que la vie d'ici-bas n'est que jeu, frivolité, parure, rivalité d'orgueil entre vous, joutes sur la quantité de biens ou le nombre d'enfants. À la semblance d'une ondée : la végétation qu'elle fait naître charme les dénégateurs, puis elle s'affole et tu la vois jaunir, et puis elle tombera en détritus. Il y aura dans la vie dernière châtiment terrible, indulgence de Dieu et son contentement. Quant à la vie d'ici-bas, elle n'est que jouissance d'illusion.» 
moderne. À la condition, bien sûr, de ne pas négliger la diversité des cultures qui ont labouré une terre présentée au sens propre et au sens figuré comme le grenier de la Méditerranée. De tous les apports qui constituent le pays, la conquête arabe au moyen âge, le développement de la langue arabe et de l'Islam occupent une place essentielle que personne ne peut - et ne veut - sérieusement contester. En réactivant un mythe préislamique, les deux romanciers ont construit un modèle littéraire fondé sur un éloge serein de la continuité. Si les identités peuvent être meurtrières, l'antidote réside dans la diversité des héritages. La Tunisie, dans ce qu'elle a de meilleur, a magnifiquement intégré - et pas seulement pour des raisons touristiques! - les ruines de Carthage, le bel amphithéâtre d'El Jem, les sites de Sbitla, de Dougga et de Thala... L'islam tunisien, du moins à l'époque moderne, n'a jamais cherché à faire table rase de cet héritage de pierres. Les deux romanciers fascinés par Elissa proposent de remonter encore plus loin dans le temps, de remonter bien avant la constitution de l'empire romain, à une époque et en des lieux où la société n'était pas encore exclusivement fondée sur le pouvoir masculin, où la séparation des sexes ne valait pas encore comme la différence fondamentale à partir de laquelle se structure la société. Donner la parole à une héroïne mythique, c'est moins pour eux exalter le pouvoir d'une femme que rappeler l'existence, plus ou moins idéalisée, d'une société où hommes et femmes pouvaient chanter et danser ensemble devant les couleurs de la mer. 